\title{
Aristotelismo, Medicina antigua y universidad mexicana.
}

\author{
Historia de la Medicina
}

Raúl Vallejos*.

Santa Fé, Argentina.

\section{RESUMEN.}

La Medicina tal como era enseñada en las primeras universidades americanas de los tiempos de la colonia y particularmente en la Real y Pontificia Universidad de México, tenía una fuerte vinculación con los principios naturales y biológicos sustentados por Aristóteles. En el siglo XVI, la enseñanza de la Medicina se realizaba en la Universidad y la carrera constaba de cuatro cursos, con un total de cuatro años, en los cuales se disponía el uso para la enseñanza del libro de Aristóteles, intitulado DE GENERATIONE ET CORRUPTIONE. El grado que se recibía después de haber realizado esos estudios, era el de bachiller en Medicina. La enseñanza de la Medicina estaba configurada por los trabajos de Hipócrates y Galeno, pero la inclusión de la obra de Aristóteles, indica que se estimaban los principios biológicos sostenidos por el Estagirita, y con ello, la aceptación de la existencia de un espíritu vital o pneuma, que animaba todos los fenómenos de la vida. El aristotelismo, como doctrina filosófica se afirma durante la Edad Media y posteriormente prevaleció en la enseñanza de las nuevas

* Publicación postuma. Reproducida de: Revista de la Universidad de Yucatán 1964; (35):99-103.
Universidades del período colonial. El aristotelismo imperó durante muchos siglos y predominó en las enseñanzas dadas en las nuevas universidades coloniales. (Rev Biomed 2001; 12:138-141)

Palabras clave: Historia de la Medicina, enseñanza médica, Aristotelismo.

\section{SUMMARY.}

Aristotelism, old Medicine, and the mexican university.

During the colonial era, the teaching of Medicine, particularly at the Real y Pontificia Universidad de Mexico, was strongly connected to the natural and biological principals held by Aristoteles Medicine was taught at the university during the sixteenth century, it was composed of four courses lasting a total of four years in which the book "DE GENERATIONE ET CORRUPTIONE" by Aristoteles was taught. After having successfully completed these studies, students were awarded a Bachelor's Degree in Medicine. The teaching of medicine was based on the work of Hipocrates and Galeno but the

Solicitud de sobretiros: M.C. Renán A. Góngora-Biachi, Centro de Investigaciones Regionales "Dr. Hideyo Noguchi”, Ave. Itzáes No 490 por 59, C.P. 97000, Mérida, Yucatán, México. Correo electrónico: gbiachi@tunku.uady.mx Recibido el 2/Mayo/2001. 


\section{$\boldsymbol{R}$ Vallejos.}

inclusion of the book by Aristoteles indicates that his biological principals were highly estimated and with that the acceptance of a vital spirit or pneuma which gave rise to all the phenomenum of life. Aristotelism, as a philosophical doctrine, was affirmed during the Middle Ages and then prevailed in the teaching of the new universities of the colonial period. Aristotelism reigned during many centuries and predominated in the education given in the colonial universities.

\section{(Rev Biomed 2001; 12:138-141)}

Key words: History of Medicine, medical education, Aristotelism.

Resulta un hecho comprobado frente a los textos utilizados y los diversos comentarios sobre los mismos, que la Medicina tal como era enseñada en las primeras universidades americanas de los tiempos de la colonia y particularmente en la Real y Pontificia Universidad de México, tenía una fuerte vinculación con los principios naturales y biológicos sustentados por Aristóteles.

Al respecto, recordemos que, por el siglo XVI, la enseñanza de la Medicina se realizaba en la Universidad y la carrera constaba de cuatro cursos, con un total de cuatro años, en los cuales se disponía el uso para la enseñanza del libro del Estagirita, intitulado DE GENERATIONE ET CORRUPTIONE, que reviste el carácter de una obra de Biología y no concretamente del campo de la ciencia médica.

En su oportunidad hemos mencionado el hecho de que la fecunda cultura mexicana, tiene el carácter de una auténtica precursora en toda la América Hispana, en lo concerniente a la enseñanza de la Medicina, por haber aparecido en la capital de la nación azteca, tres libros de medicina escritos por el doctor en Medicina Francisco Bravo, Fray Agustín Farfán y Alonso López de Hinojoso.

Por otra parte, esas obras, aparecidas todas ellas en el transcurso del siglo XVI, contienen agudas observaciones anatómicas y clínicas, como asimismo, acertadas medicaciones, o prescripciones terapéuticas con la presencia de los inevitables prejuicios de la época.

Indudablemente, desde la fundación se enseñó con preferencia Prima de Teología y Vísperas de Teología, Cánones y Leyes (Prima de Derecho Civil) y posteriormente se enseñó Medicina. Como es de conocimiento general, la Real y Pontificia Universidad, desde el año de 1578, contaba ya con su cátedra de Medicina. Normalmente las clases se impartían en horas de la mañana y por lo general, las tesis se rendían después de las quince horas. El grado que se recibía después de haber realizado esos estudios, era el de bachiller en Medicina.

La enseñanza de la Medicina estaba configurada por los trabajos de Hipócrates y Galeno, pero la inclusión de la obra de Aristóteles intitulada DE GENERATIONE ET CORRUPTIONE, indica que se estimaban los principios biológicos sostenidos por el Estagirita, y con ello, la aceptación de la existencia de un espíritu vital o pneuma, que animaba todos los fenómenos de la vida. Asimismo, eran obligatorios los textos DE LOS APHORISMOS, DE LOS PRONOSTICOS Y DE LAS EPIDEMIAS de Hipócrates y del ARS PARVA de Galeno y posteriormente se utilizó el CANON de Avicena.

De esta manera, los principios biológicos sostenidos por Aristóteles, se mezclaban en la enseñanza con los trabajos de Hipócrates, Galeno y Avicena. El curso incluía la enseñanza de la Cirugía y Medicina y se encerraban dentro de un empirismo elemental, los principios de Hipócrates y Galeno; la Medicina especulativa con la Filosofía Natural del Estagirita; la Biología con la Patología humoral, y la vis medicatrix natural con la medicación de Avicena. Con todo ello se advierte que la influencia de Aristóteles en el campo médico, se extendió durante varios siglos.

Una de las características impuestas por el propio fundador del Liceo, en sus especulaciones médicas, es la búsqueda de las causas que contribuían a la presencia de la salud o bien de la enfermedad. Con ello se intentaba fijar ciertas

\section{Revista Biomédica}


Aristotelismo en la Medicina Antigua.

normas, para establecer el desequilibrio que provocaba una determinada afección.

Como es de conocimiento del lector, el Estagirita no escribió obra alguna dedicada exclusivamente a la Medicina, pero tenía conocimientos muy certeros acerca de la Anatomía en general, Anatomía comparada, Biología, Embriología, Epidemiología e Higiene. Hace muy claras descripciones clínicas de los síntomas de algunas enfermedades y de los climas más adecuados para el desarrollo de la existencia humana. Hay afirmaciones erróneas, pero ello no invalida en forma alguna, su admirable aporte científico en el campo de la Biología.

Como los grandes médicos de la antigüedad, valora mucho la alimentación apropiada para el sostenimiento de la salud y defiende a la dietética para dominar a la enfermedad en el organismo humano.

Por su parte, los defensores de Hipócrates, confiaban en la vis medicatrix naturae o sea el poder curativo de la naturaleza, que también en principio aceptaba el propio Estagirita, al referirse a la Dietética como la forma más efectiva de la terapia, tratando de limitar todo lo posible el suministro de medicamentos.

Tomada en su conjunto la enseñanza impartida en las antiguas universidades americanas, la Medicina con cuyos estudios completos, se obtenía el título de Bachiller en esa ciencia, tenía un carácter marcadamente deductivo y mantenía una filosofía especulativa acerca de los elementos humoroles, que configuraban la existencia de la salud. Se la impartía a través de los textos de Aristóteles, Hipócrates y Galeno, aunque también se enseñaba la Cirugía y la Anatomía.

Con todo ello, la enseñanza se resentía por tener una modalidad abiertamente verbalista. Y de esta manera, se repetían errores tradicionales, tales como que el corazón tenía tres ventrículos, como lo había establecido Aristóteles. Como es sabido, en cierta manera ese autor, era de tendencia hipocrática, pero supera ampliamente al fundador de la Escuela de Cos, por sus amplios conocimientos en Anatomía normal, Anatomía en general y Anatomía comparada.

Los conocimientos anatómicos de los hipocráticos, no eran ni muy certeros, ni muy profundos, todo lo contrario que ocurrió con Aristóteles, quien realmente es el fundador de la Anatomía comparada y de la Biología, tuvo atisbos geniales.

Con todo, la enseñanza de la Medicina en la Real y Pontificia Universidad de México, se encerraba en los moldes tradicionales de los conceptos de Aristóteles, Hipócrates y Galeno. Resulta innegable que el Estagirita, tenía conocimientos clínicos sobre las enfermedades y sobre la dieta para mantener el equilibrio biológico de la salud. Ello configura indudablemente una Medicina empírica, en la cual predominaba: una enseñanza con base de textos clásicos y la aplicación muy limitada de la observación directa.

$\mathrm{El}$ aristotelismo, como doctrina filosófica se afirma durante la Edad Media y posteriormente prevaleció en la enseñanza de las nuevas Universidades del período colonial. Por ello, los principios biológicos y la presencia de causas naturales y no naturales, se enseñaron en los cursos de Medicina, así como no faltaron los libros de Metafísica, para la enseñanza de la filosofía. Las obras de Biología del Estagirita, configuraron principios que se utilizaron por ese entonces, en el campo de la Medicina.

Con todo lo dicho, se advierte que el aristotelismo imperó durante muchos siglos y predominó en las enseñanzas dadas en las nuevas universidades coloniales. En esa enseñanza, como en el desarrollo de la medicina griega del siglo $\mathrm{V}$, antes de Cristo, se le daba gran importancia a la dieta, cuya significación ya señalaba en sus libros el propio Aristóteles.

Concretamente, el fundador del Liceo, se imponía también al aparecer como el fundador de la Anatomía comparada, en lo cual supera innegablemente a los conocimientos de los representantes de la escuela hipocrática. De esta manera se explica su aceptación universal y que se

Vol. 12/No. 2/Abril-Junio, 2001 


\section{1}

\section{$\boldsymbol{R}$ Vallejos.}

lo defendiera aún en sus propios errores.

Todo esto nos explica el predominio del aristotelismo y la difusión de sus trabajos, desde el comienzo de la enseñanza, en la cátedra de Medicina de la Real y Pontificia Universidad, desde el año de 1578, es decir, ya por la segunda mitad del siglo XVI.

En consecuencia, el progreso de los conocimientos médicos fue lento y solamente a través de largos años de enseñanza, la misma tuvo el carácter de experimental y se realizó sobre observaciones directas.

Se reconoce, que ya por el siglo XIII, se practicaba la cirugía en la época medieval, empero, en la enseñanza en tierras americanas, se realizaron muy contadas disecciones.

De esta manera, se demuestra el predominio del pensamiento antiguo y la preponderancia del aristotelismo, al cual se agregó más tarde en la enseñanza médica, el CANON, vierte importantes observaciones anatómicas, clínicas y fisiológicas, aportando numerosas indicaciones acertadas para el tratamiento de las enfermedades.

Ibn Sina o Avicena (880-1037), eximio representante de la ciencia árabe, se interesó asimismo por la cirugía y trajo un soplo renovador en el campo de la Medicina, que posteriormente llegaría a las tierras americanas. Por otra parte, Avicena, tenía verdadera estimación hacia Aristóteles, por sus admirables trabajos acerca de la Biología, la Anatomía comparada y la Embriología.

Luego, con el paso del tiempo, fueron enseñadas las modernas concepciones del notable anatomista Andrés Vesalio y del renombrado cirujano Ambrosio Paré, que transforman los conocimientos y traen principios renovadores para las aulas de las antiguas universidades americanas, aportados desde la Madre Patria, donde ya se habían constituido distintas escuelas médicas de evidente significación.

\section{Revista Biomédica}

